${ }^{\bullet}$ Entomologica Fennica. 29 June 1994

\title{
Freeze-drying techniques for preserving aphids (Homoptera, Aphidodea)
}

\author{
Anders Albrecht
}

Albrecht, A. 1994: Freeze-drying techniques for preserving aphids (Homoptera, Aphidodea). - Entomol. Fennica 5:105-113.

Techniques for collection, preparation and storage of freeze-dried aphid samples, including galls, are described. Freeze-drying can be done with the aid of a home freezer, a drying agent, and suitable containers alone, but drying time can be reduced considerably with cheap and simple vacuum drying equipment. Freeze-drying methods have several advantages compared with traditional mounting techniques. Body shape, colours, wax coating and microsculpture are excellently preserved. The labour required per sample, for preparation as well as for identification, is reduced to a minimum, and complete colony samples can be stored as entities. Aspects of practical handling and study of freeze-dried aphid samples are discussed.

Anders Albrecht, Finnish Museum of Natural History, Zoological Museum, P.O. Box 17, FIN-00014 University of Helsinki, Finland

Received 1 August 1993, accepted 20 October 1993

\section{Introduction}

Aphid samples for identification and taxonomic purposes are traditionally mounted on microscope slides, which requires treatment of the specimens in a series of different chemicals (e.g. Heie 1980, Danielsson 1985, Eastop \& van Emden 1972, Heikinheimo 1988). Techniques for freeze-drying caterpillars have been described by among others Flaschka \& Floyd (1969) and Dominick (1972), whose papers include useful technical and practical information on drying agents and vacuum equipment as well as drying times. Freeze-drying has not, however, previously been used as a routine method for preserving aphid samples.

The conventional preservation methods have a number of shortcomings: 1) Colour characters except dark pigmentation are lost and any wax patterns are destroyed, 2) the body of the speci- men becomes more or less distorted, and important characters may be fixed in positions that make them difficult to study, 3) reticulation and other microsculpture is considerably weakened, or even obliterated, 4) the preparation of slides is time-consuming and requires continuous attention, and (5) slides can accomodate only a few specimens each, which leads to split colony samples and usually to restricted numbers of specimens in museum collections.

The methods described in this paper aim at minimising these shortcomings and are intended to serve as a general preservation and storing system, allowing Macro and SEM photography as well as mounting on slides when necessary. Identification, drawing and measuring can be done on freeze-dried specimens directly, and the method minimises the need for making field notes on colour and wax characters. 


\section{Collecting}

The methods for collecting aphid samples intended for freeze-drying are largely the same as those conventionally employed, the main difference being that the specimens should if possible be brought to the laboratory alive, because aphids deteriorate rapidly after death. On longer expeditions, and when further breeding of the material is intended, the aphids should be collected with part of the host plant, and preferably not removed from their feeding site. Petri dishes or glass or plastic vials large enough to accommodate the colonies are suitable for this purpose. In order to absorb honeydew droplets and excess moisture, the containers should be lined with tissue paper. Exposure to direct sunlight or extreme temperature fluctuations may be disastrous for the samples. Ants remove honeydew very efficiently from ant-attended colonies, and normally continue doing so even in small containers. Care should, however, be taken not to disturb the ants, as they otherwise may kill the aphids by exuding large amounts of acid. Syrphid larvae and other predators should be removed from the colonies in the field, or they will rapidly destroy the samples.

Colonies can be kept alive for several days or even weeks, provided that moulded and decayed plant parts are replaced with fresh ones. On short expeditions it is convenient to remove the aphids from the host plant and collect them directly in the containers intended for the drying process (see below).

The methods for handling live aphids depend largely on colony structure and on the aphids themselves. Specimens with wax wool or a thick wax pattern are to be handled extremely gently, preferably with a soft hair brush. Less delicate aphids can conveniently be handled with tweezers, brushes or suction devices.

As the time and labour required for preservation is indifferent to sample size, large samples containing all morphs and stages should be preferred. Ants, predators and parasites found in or reared from the aphid colonies should not be thrown away. Soft-bodied predators such as syrphid and coccinellid larvae can successfully be freeze-dried using the same methods as those for aphids. Freeze-dried galls are extremely informative, as they keep their colour and shape.

\section{Killing and drying}

Unless legs and antennae are to be positioned, the aphids are best put into the freeze-drier alive. If dead material is needed, ethylacetate fumes or some other relaxing poison is suitable. Alcohol must not be used, as it prevents the material from freezing.

The freeze-drying equipment need be neither expensive nor elaborate. A freezer, sample vials, an air-tight container and silica gel or some other desiccant is all that is needed. Vacuum equipment (see below) reduces drying time considerably, but is in no way necessary.

I shall first describe the simplest freeze-drying system, and then move on to more elaborate and efficient methods. The directions below pertain to a 1 litre cubic container, just to give a rough idea of how the system works. The container need not be absolutely air-tight.

1. The container with a $2-3 \mathrm{~cm}$ thick layer of dry (blue) silica gel on the bottom is cooled well below the freezing point, preferably below $-15^{\circ} \mathrm{C}$, in the freezer.

2. To ensure that the aphids extend their rostrum, they should, unless in situ colony samples are desired, be removed from their host plant. The aphids are put into test tubes or other suitable vials lined with tissue paper, and the tubes closed with loose cotton wool stoppers or, better, with thin, close fabric, e.g. chiffon. If cotton wool stoppers are used, they should also be lined with tissue paper, as the aphids otherwise may become entangled in the wool. In order to allow close contact between the aphids and the desiccant, short, wide vials are preferable to ordinary test tubes. Plastic vials $1 \mathrm{~cm}$ tall with a gauze bottom are excellent.

3. The vials with the aphids are put upon or, better, embedded in the silica gel layer (filled up with frozen gel if necessary) and the container closed and left in the freezer.

4. When the aphids are suspected to be dry, a few samples can be taken out and inspected under the stereo microscope. When the specimens are completely dry, their surface is mat, and no dark or translucent patches can be seen. Ice inside the body will rapidly melt 
under the microscope lamp and appear as moisture on the surface. Specimens that are not completely dry can usually be saved if they are immediately frozen again. It is always safer to leave the material in the freezer for some time after minimum drying time has expired. Until processed further, dried specimens are best kept in air-tight containers with some desiccant.

Drying time is dependent on several factors. Higher temperature speeds up the drying process, but in order to avoid thawing of the samples when the freezer lid is opened, temperatures well below $-10^{\circ} \mathrm{C}$ are recommended.

Low air humidity around the samples likewise shortens drying time. To ensure that the water evaporating from the samples is removed as effectively as possible, the sample vials should be arranged so that the distance between the aphids and the desiccant is kept at a minimum. And, naturally, the duration of drying is dependent on the size and cuticle of the aphids.

As a rule of thumb, if the above recommendations are observed, the aphids should dry in an ordinary food freezer at about $-18^{\circ} \mathrm{C}$ completely in two or three months.

More samples can be added to the container at any time, but thawing of samples already in the container must be avoided. The vials and containers with deep-frozen aphids must be handled very gently. Before the aphids are dry their legs and antennae are extremely brittle. Dry samples, on the other hand, can stand rough handling surprisingly well.

If the silica gel becomes moist (red) during the process, it should be replaced with cold dry gel. The desiccating properties of used silica gel can be regenerated by heating it in an electric oven at $150-200^{\circ} \mathrm{C}$ until its deep blue colour is restored.

At least for amateur aphidologists I think the method described above, without vacuum equipment, will be quite satisfactory. The rather long drying time will be of little importance, as further processing of the material is hardly likely to begin before the collecting season is over, and by that time, most of the samples will be ready.

If shorter drying times are required, vacuum equipment is recommended. With commercial

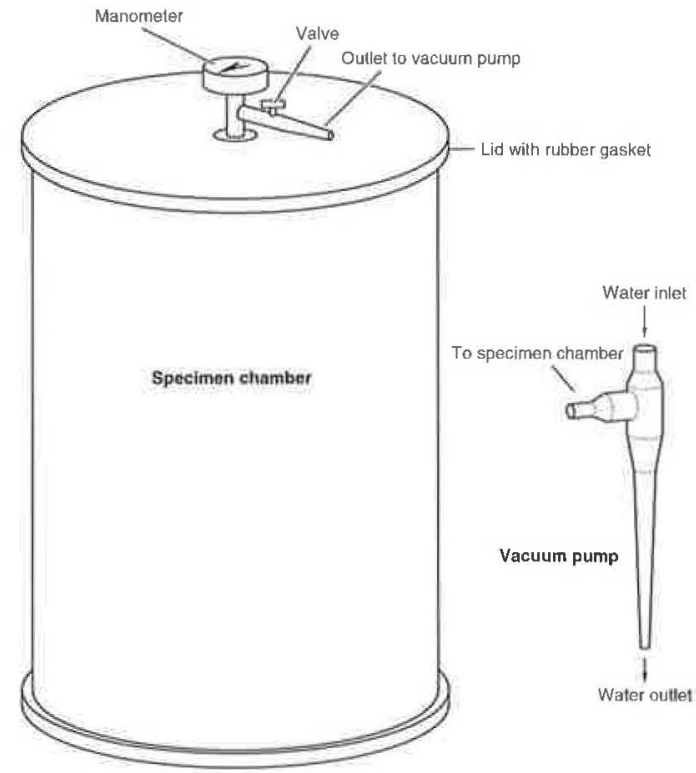

Fig. 1. Acrylic vacuum container for freeze-drying aphids and other biological specimens. Diameter 30 $\mathrm{cm}$, height $40 \mathrm{~cm}$, wall thickness $10 \mathrm{~mm}$; manometer not necessary, but most useful. This model has a capacity of 1000-2000 aphid samples in $12 \times 70 \mathrm{~mm}$ test tubes or $25 \times 10 \mathrm{~mm}$ plastic vials. Smaller containers can, for instance, be made of strong glass jars.

vacuum freeze-driers the time can be reduced to a few days or less, but the equipment is very expensive.

In order to reduce costs, I have developed a system which is cheap, is simple to use, and has proved very effective. The general procedure is similar to that described above, but the boxes (which in this case may not be air-tight) with the aphids and silica gel are placed into an air-tight, pressure-resistant chamber (Fig. 1) in the freezer, and the chamber evacuated with a water-driven vacuum pump (for systems with other types of pumps, see e.g. Dominick 1972).

The degree of vacuum depends on the water pressure, and drying time can be considerably reduced by connecting the water inlet of the pump to the outlet of a high-pressure washer (make sure the pipes and junctures can stand the pressure). The latter alternative also guarantees constant water pressure, which is important because fluctuations may allow humid air to flow towards 
the specimen chamber and cause the valve and pipes to freeze. After evacuation, the outlet valve of the chamber can be closed and the pump disconnected.

With the system described in Fig. 1 and a water pressure of 30-40 bar and a temperature of $-18^{\circ} \mathrm{C}$ in the specimen chamber, maximum vacuum is reached after about 15 minutes. The vacuum obtained is sufficient for most samples to dry within a week or two, and some specimens may be dry even after a couple of days. Because of the variation in drying time between aphid individuals, the samples are better kept in the chamber for a month or so. When the container is to be opened for removal or addition of samples, the air pressure should be raised very slowly, or the specimens may shrink.

Some specimens will not dry properly at all, probably because their body fluid composition is such that it will not freeze. Such specimens may occur in any colony and particularly among young and half-grown nymphs. Normally, however, most specimens in a colony will dry without problems.

The drying techniques can be applied to galls as well. The drying time is about the same as for aphids, provided there is enough silica gel. In order to save storage space, larger galls can be lightly pressed between pieces of cardboard. Excessive pressure can be prevented by placing small pieces of foam polystyrene or other suitable material between the cardboard pieces.

\section{Storage}

If freeze-dried aphid samples are stored in test tubes, the specimens are inclined to roll about, which may cause damage to legs, antennae and, above all, to the wax coating. Because the optical properties of the tube walls makes microscopy difficult, the specimens have to be taken out for study. A system by which samples can be safely stored and easily studied under the microscope with a minimum of handling, and which has proved practical and efficient, is described below.

The sample storage unit consists of a foam polystyrene plate with a shallow circular specimen chamber and a tightly-fitting lid of trans-

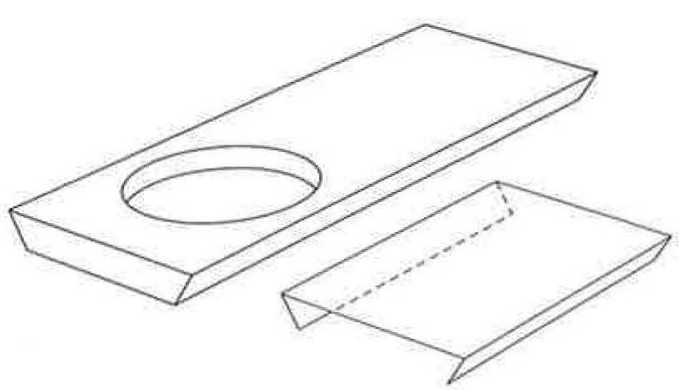

Fig. 2. Container for storage of freeze-dried aphids, made of dense foam polystyrene, with a lid of cellulose acetate film. Excavation drilled with an electric router (milling machine) at $25000 \mathrm{rpm}$, producing smooth and even surfaces. Dimensions: length $75 \mathrm{~mm}$, width $32 \mathrm{~mm}$, thickness $6 \mathrm{~mm}$, excavation diameter $25 \mathrm{~mm}$, depth $4 \mathrm{~mm}$.

parent cellulose acetate film (e.g. overhead projector film), Fig. 2. The chamber is large enough to accommodate colony samples of most aphid species. For the largest species it may be necessary to deepen the excavation, and very large samples can be stored in larger containers, or the samples divided into several units. Some space can be saved by using smaller plates for samples containing only one or a few specimens, but generally a standardised size is more practical.

Before samples are put into containers it is wise to remove sand and litter particles and specimens that have failed to dry properly. This is best done by pouring the sample into a petri dish where aphids can be separated from unwanted material with a fine brush. The debris can then be removed with a suction device, and the aphids poured into the chamber.

The lid can be closed either by bending it upwards and applying it from above, or by sliding it sideways over the excavation. Newly manufactured plates and lids tend to be charged with static electricity, which may cause some difficulties.

Labels with collecting and identification data can be glued to the plates with water-soluble joiner's glue. Bubbles can be avoided by soaking the labels in water before applying the glue.

For storage of sample plates I use trays made of foam polystyrene (Figs. 3 and 4A) which I 


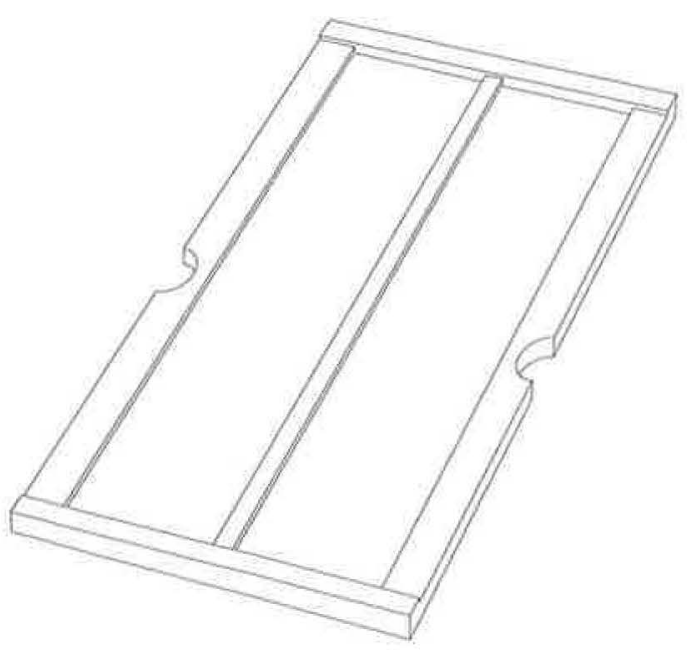

Fig. 3. Collection tray for storage of freeze-dried aphid samples, made of dense foam polystyrene. Dimensions of parts: bottom plate $297 \times 210 \times 6 \mathrm{~mm}$ (A4 paper format), front and rear bars $210 \times 10 \times 6 \mathrm{~mm}$, mid bar $277 \times 10 \times 3.5 \mathrm{~mm}$, side bars $277 \times 21 \times 3.5 \mathrm{~mm}$, incision diameter $25 \mathrm{~mm}$. Parts joined with watersoluble joiner's glue before lateral incisions are drilled.

keep stacked on top of each other. The trays are dimensioned so that microscope slides can be stored along with the freeze-dried samples, and are thick enough to allow labels to be glued onto the front vertical surface.

Freeze-dried samples stored in this way, if handled with care, are fairly insensitive to mechanical damage, and require less space per specimen than do traditional slide collections; individual samples are easy to locate. Compared with slide collections, however, freeze-dried material requires more attention. Although the containers offer good protection against dermestid larvae and other injurious insects, they cannot be guaranteed to be absolutely pest-proof.

Type specimens could, for maximum safety, be glued onto a piece of paper, e.g. with Euparal, in such a way that no unique (unpaired) characters are obscured. The paper can be fastened to the chamber bottom with a micro pin.

For storage of galls, I use boxes $1 \mathrm{~cm}$ high with walls of foam polystyrene, a bottom of paper or cardboard, and lid of cellulose acetate film fastened with short pins in the corners.

\section{Identification and study}

Routine identification of most material can be carried out with an ordinary stereo microscope without removing the specimens from the containers, in many instances even without opening the lids. Characters such as colour, shape, general pilosity, sclerotisation, wax pattern and so forth are readily visible. Coarse measurements can be taken, and reticulation and other microsculpture can generally be studied without difficulty. Most of the characters illustrated in Figs. 4-6 can easily be studied without further equipment.

More detailed studies require a high-power stereo microscope or a transmission microscope with arrangements for incident light. For ordinary light microscopes, this arrangement allows magnifications of up to 200 times or more without crushing the specimens. Generally, transmission microscopes have better optical properties than stereo microscopes also at lower magnifications. Most measurements can be taken and such items as hairs and rhinaria counted without making slide preparations.

If positioning is required, as for drawing, the specimens can be supported by micropins either in the containers or on pieces of foam plastic, which are then rotated into the desired position, or glued to pieces of paper or cardboard.

In addition to colour, shape and wax pattern, reticulation and other microsculpture characters are much better preserved with freeze-drying than with traditional slide-mounting techniques. This means that they can be studied in greater detail and at lower magnifications, both as structures and as the general impressions they give, such as different degrees of matness and iridescence. Slides of detached legs and antennae can successfully be made without elaborate degreasing and clearing procedures. Just softening in hot water or lactic acid is sufficient. And, if needed, freeze-dried specimens can successfully be cleared and mounted on slides and studied in the traditional manner. For SEM studies freeze-dried samples are ready as such.

The internal body colour of many aphids will fade to a lighter shade as specimens dry and the body fluid is replaced with air. This colour change is particularly striking in black aphids such as 


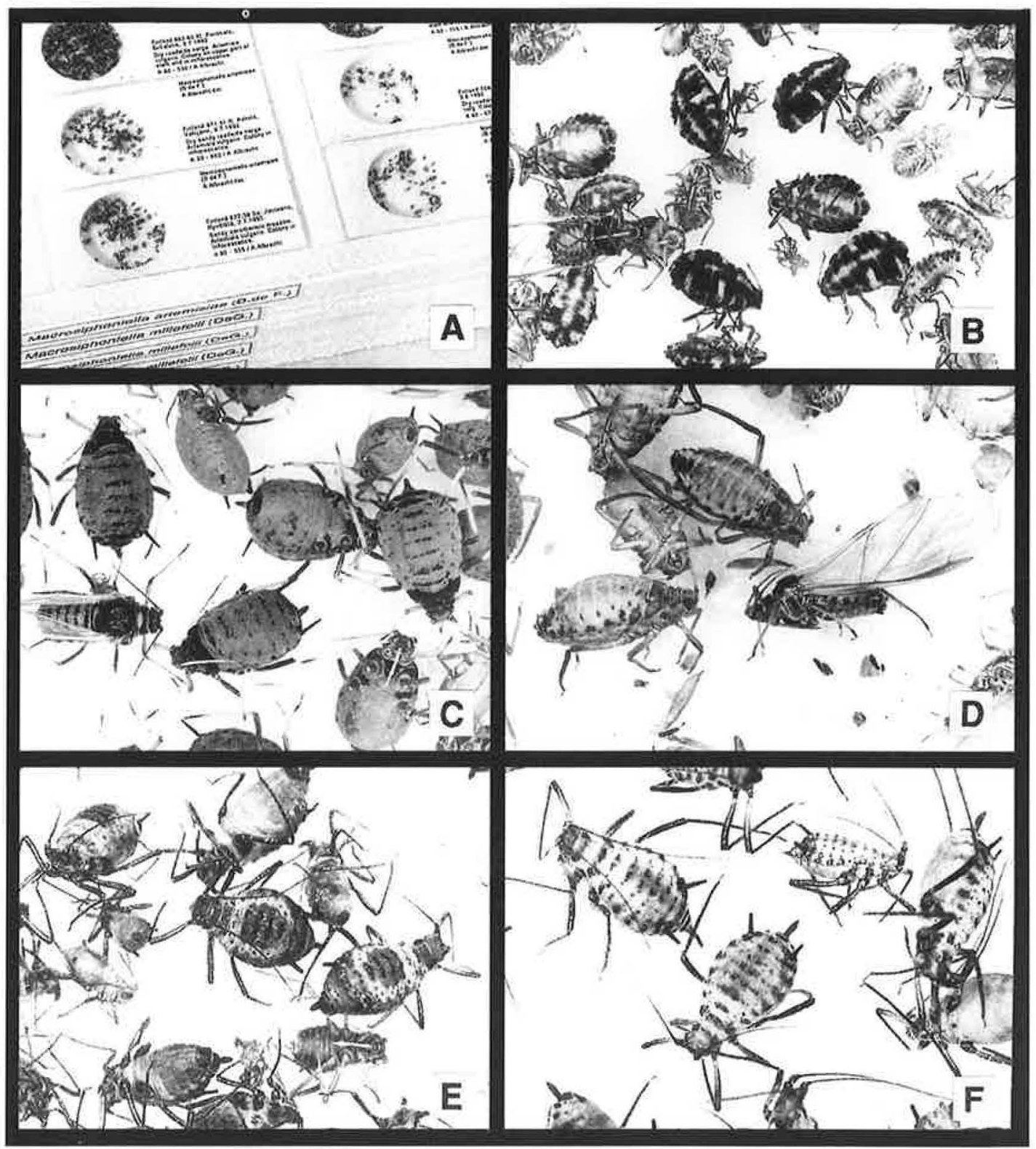

Fig. 4. Freeze-dried aphids. A: Stack of collection trays with sample containers: Macrosiphoniella artemisiae (Boyer de Fonscolombe) and M. millefolii (DeGeer). B-F: Macro photographs of samples, showing colour and wax patterns, and sclerites. B: Glyphina sp. (apparently G. pseudoschrankiana Blackman, from Betula pendula). The light markings are due to weaker sclerotisation, not to wax (see also Fig. 6A-B). C: Aphis fabae cirsiiacanthoidis (Scopoli). D: Pterocomma pilosum konoi Hori ex Takahashi. E: Macrosiphoniella absinthii (Linnaeus). F: M. millefolii. Nomenclature in Figs. 4-6 after Eastop \& Hille Ris Lambers (1976).

Aphis fabae (Scopoli) and Pterocomma salicis Linnaeus. The fading makes the sclerotic pattern and colour shades stand out distinctly. Wax patterns, on the other hand, become less distinct, al- though still clearly visible. The original black can be restored by soaking the specimens in water.

Some colours are irreversibly lost or changed in the drying procedure. The pink tinge of 

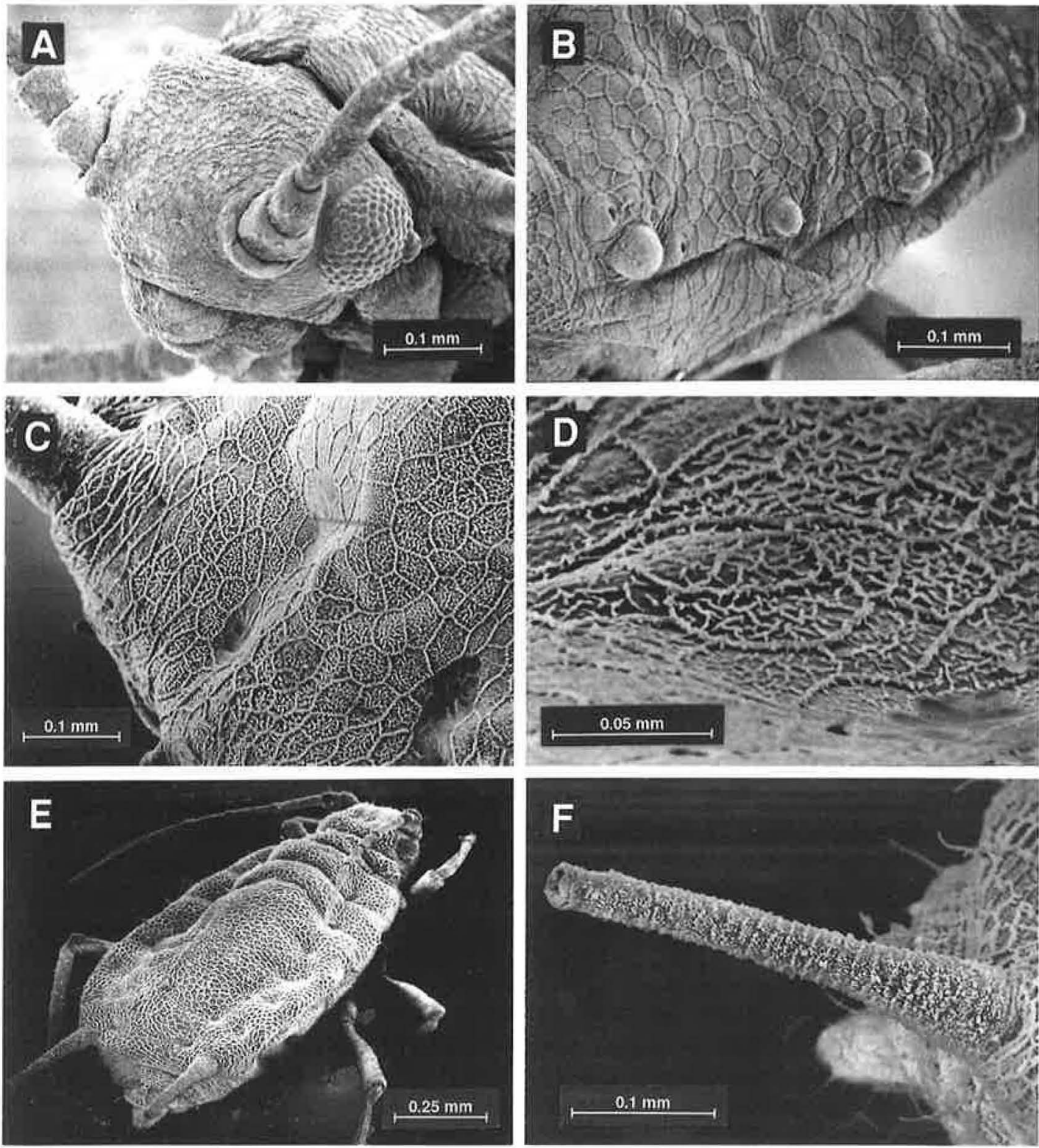

Fig. 5. SEM micrographs of freeze-dried aphids. Apterous viviparous females. A-B: Aphis klimeschi (Börner), head and abdomen, showing wax gland reticulation and lateral tubercles. C: Aphis fabae cirsiiacanthoidis (Scopoli), presiphuncular wax gland reticulation. D: Part of the same enlarged in oblique lateral view. E: Aphis idaei van der Goot, showing wax reticulation. F: Siphunculus of the same, showing cuticular imbrication and wax granulation.

Metopeurum fuscoviride Stroyan vanishes almost completely, whereas other reddish colours seem to be permanent (e.g. in Uroleucon tanaceti (Linnaeus) and the eyes of Macrosiphoniella species). In Aulacorthum the green and yellowish almost invariably change to dirty brown or brownish yellow. Green colours in other genera are well preserved in the drying process, but fade to pale yellow with time.

The methods for reconstitution of dry specimens for dissection or histological studies presented by Van Cleave \& Ross (1947) and Harris 

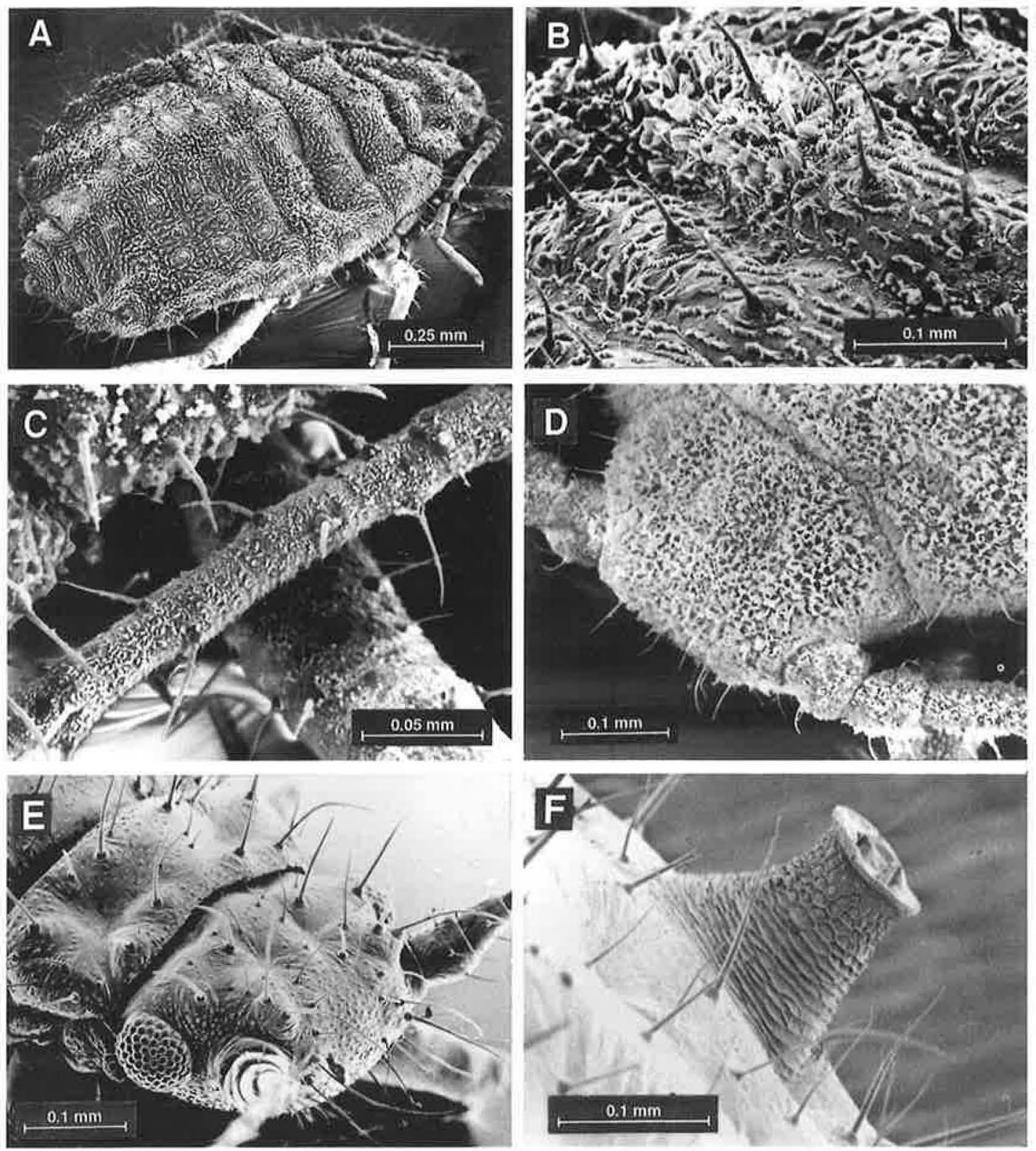

Fig. 6. SEM micrographs of freeze-dried aphids. Apterous viviparous females. A: Glyphina ?pseudoschrankiana (see Fig. 4), showing chaetotaxy and distinct wax pattern. Note the areas of rich wax production on the light markings of the cuticle (see Fig. 4B). B: The same, wax patch on abdominal tergite 1. C: The same, third antennal segment with wax granulation. D: Geoica setulosa (Passerini), head with thick reticulate wax bloom. E: Chaitophorus populialbae (Boyer de Fonscolombe), head and prothorax, showing chaetotaxy and cuticular granulation. F: $C$. populeti (Panzer), siphunculus with cuticular reticulation.

(1964) can most certainly be applied to freezedried aphids as well.

When using keys and handbooks, it should be kept in mind that some characters of freeze- dried specimens are not directly comparable with descriptions based on slide-mounts. Microsculpture, particularly reticulation in conjunction with wax secretion, may be completely lost in 
slides (see for example Fig. 5E: according to Heie (1986), Aphis idaei has no reticulation at all), or it is visible only on the sclerotised parts or with phase-contrast, even if it is very distinct in freeze-dried material. The pressure exerted by the coverslip on different body parts causes variable degrees of distortion. Particularly susceptible to this distortion are body shape and length, siphuncular width, shape of the frontal tubercles, and the shape of the rostrum which becomes more or less flattened under the body.

\section{Mailing material}

Mailing freeze-dried aphid samples is far less safe than mailing slide mounts. Because of their fragility the dried specimens must be prevented from shaking around during transportation. Even if the sample containers normally can be dropped to the floor without any damage happening to the specimens, I would be reluctant to mail samples without special precautions. The aphids can fairly effectively be protected from mechanical damage by loosely filling the empty space of the chamber with pappus hairs of dandelion (Taraxacum) or some other suitable plant. Another possibility is to use fine fibres, e.g. from nylon cloth.

Acknowledgements. I wish to thank my friends Osmo Heikinheimo, Larry Huldén and Lauri Kaila for encouragement and valuable comments, and Reino Tyynelä for letting me use his photo laboratory. My special thanks are due to Matti Viitasaari for his help and patience during my work with the SEM microscope at the Department of Applied Zoology, University of Helsinki. I am also indebted to Carol Norris, who kindly checked the English of the manuscript.

\section{References}

Danielsson, R. 1985: Polyviol as a mounting medium for aphids (Homoptera: Aphidoidea) and other insects. Entomol. Scand. 15:383-385.

Dominick, R. B. 1972: Practical freeze-drying and vacuum dehydration of caterpillars. - J. Lepidopterol. Soc. 26:69-79.

Eastop, V. F. \& Hille Ris Lambers, D. 1976: Survey of the World's aphids. — Junk, The Hague. 573 pp.

Eastop, V. F. \& van Emden, H. F. 1972: The insect material. - In: van Emden, H. F. (ed.), Aphid technology: 1-45. Academic Press, London and New York. 344 pp.

Flaschka, H. A. \& Floyd, J. 1969: A simplified method for freeze-drying caterpillars. - J. Lepidopterol. Soc. 23:43-48.

Heie, O. 1980: The Aphidoidea (Hemiptera) of Fennoscandia and Denmark. I. - Fauna Entomol. Scand. 9:1-236.

- 1986: The Aphidoidea (Hemiptera) of Fennoscandia and Denmark. III. - Fauna Entomol. Scand. 17:1314.

Heikinheimo, O. 1988: Mounting techniques, aphid collections. - In: Minks, A. K. \& Harrewijn, P. (eds.), Aphids, their biology, natural enemies and control: 31-44. - World crop pests 2B. Elsevier Science Publishers, Amsterdam, Oxford, New York and Tokyo. 364 pp. 